\title{
Classification of robotic battery service systems for unmanned aerial vehicles
}

\author{
Tien $\mathrm{Ngo}^{1 *}$, Mehmet Guzey ${ }^{2}$ and Vladimir Dashevsky ${ }^{3}$ \\ ${ }^{1}$ SUAI, Department of Electromechanics and Robotics, 190000, Sankt-Petersburg, Bolshaya Morskaya Str., 67, Russia \\ ${ }^{2}$ Erzurum Technical University, Department of Electrical and Electronics Engineering, 25700, Erzurum, Turkey \\ ${ }^{3}$ SPIIRAS, Laboratory of Autonomous Robotic Systems, 199178, Sankt-Petersburg, 14-th line of V.I., 39, Russia
}

\begin{abstract}
Existing examples of prototypes of ground-based robotic platforms used as a landing site for unmanned aerial vehicles are considered. In some cases, they are equipped with a maintenance mechanism for the power supply module. The main requirements for robotic multi-copter battery maintenance systems depending on operating conditions, required processing speed, operator experience and other parameters are analyzed. The key issues remain questions of the autonomous landing of the unmanned aerial vehicles on the platform and approach to servicing battery. The existing prototypes of service robotic platforms are differed in the complexity of internal mechanisms, speed of service, algorithms of joint work of the platform and unmanned aerial vehicles during the landing and maintenance of the battery. The classification of robotic systems for servicing the power supply of multi-copter batteries criteria is presented using the following: the type of basing, the method of navigation during landing, the shape of the landing pad, the method of restoring the power supply module. The proposed algorithmic model of the operation of battery power maintenance system of the multi-copter on ground-based robotic platform during solving the target agrarian problem is described. Wireless methods of battery recovery are most promising, so further development and prototyping of a wireless charging station for multi-copter batteries will be developed.
\end{abstract}

\section{Introduction}

Automation of the process of recovering the energy resources of the multi-copters on the ground-based robotic platform in the target solution area will allow for increased the duration of the multi-copters operation and the scope of the tasks in the autonomous mission. Increased autonomy for the operation of multi-copters in the target area by the automatic charging system is the subject of many scientific studies. There are two types of station recharging system and systems with battery replacement. Systems with battery replacement can significantly shorten the preparation time of the multicopters for a new flight and increase the total number of multi-copters that are simultaneously in the autonomous mission. The recharging system has a lower cost compared to the battery replacement system due to the minimization of the mechanical components of the structure.

The development of charging robotic ground stations is relevant for solving the problems of monitoring remote territories, in which most of the energy resources is used on reaching the specified area. In this case, multi-copters have the opportunity to replenish their energy resources in the territory of operation and work in a continuous mode until the end of the platform resources. The developing charging system should solve the problem of automating the battery maintenance process and could be used in fixed locations. In the framework of this study, models and prototypes of the battery charging system is developed, characterized by wireless transmission of energy, mechanization of the manipulation process with the battery on the multi-copter and the service platform.

Ground-based robotic systems and unmanned aerial vehicles, including multi-copters are actively used in various industries both for solving applied specialized tasks and for the entertainment industry. Considering the wide range of training of operators and functional capabilities of the multi-copters, it is necessary to distinguish three most popular applications of multicopters: 1) researchers; 2) farming; 3) security [1-3].

Table 1 lists the main requirements for service systems of multi-copter power supply systems for the three above categories [4]. The potential uses for security multi-copters are reconnaissance, target spotting, riot control and border patrol, among others. Uses for farming multi-copters are checking cattle, checking fences, monitoring crops and spraying crops, among others. Uses for researchers are recreational play, pilot training and data collecting, among others.

Different customers also have different design constraints (budget, weight, complexity), but it is possible to identify some major common constraints; they are summarized in Table 2.

\footnotetext{
Corresponding author: quoctienbn@gmail.com
} 
Table 1. Customer needs summary [4].

\begin{tabular}{|l|l|l|}
\hline \multicolumn{1}{|c|}{ Security } & \multicolumn{1}{|c|}{ Farming } & \multicolumn{1}{|c|}{ Researchers } \\
\hline 1. Charge UAV batteries without human assistance
\end{tabular}

When developing automatic battery power maintenance system of the multi-copter should also take into account the following functional and technical requirements:

- Provide identifiable landing space: 1) Provide sufficient area to land (bigger than 1.5 times the position error of navigation system and proportional to the number and size of skids/footprint/tail); 2) Provide means to communicate current position of platform to navigation solution.

- Charge batteries: 1) Provide safe electrical interface between battery on multi-copter, multi-copter electronics, charger on platform, and multi-copter detection system when appropriate on appropriate linkage; 2) Identify that multi-copter has landed in correct position; 3) Charge battery; 4) Identify charge needs.

- Provide power to the system: 1) Acquire power; 2) Adapt power to be used on the platform.

- Provide portability: 1) Provide easy setup; 2) Provide way to transport.
Table 2. Design constraints

\begin{tabular}{|c|l|}
\hline 1 & $\begin{array}{l}\text { Modifications to the helicopter, if any, should add as little } \\
\text { weight as possible to prevent reduction in flight duration } \\
\text { due to increase of body mass }\end{array}$ \\
\hline 2 & $\begin{array}{l}\text { Battery disabling systems must guarantee that the UAV } \\
\text { will not be disabled unintentionally }\end{array}$ \\
\hline 3 & UAV dimensions and physical properties \\
\hline 4 & The battery is very sensitive to recharging voltage/current \\
\hline 5 & $\begin{array}{l}\text { UAV electronics should not be connected to battery } \\
\text { during recharging }\end{array}$ \\
\hline 6 & Pilot skills/auto-pilot skills \\
\hline 7 & Human strength/skills \\
\hline
\end{tabular}

Above functional and technical requirements will be taken into account when developing a ground-based robotic platform equipped with landing sites and multifunctional mechanisms for capturing unmanned aerial vehicles (UAVs) and servicing their embedded power systems, taking into account previously developed technological solutions [5-7].

\section{Electromechanical maintenance systems for unmanned aerial vehicles}

Autonomous landing of unmanned aerial vehicles in modern studies is considered not only on a fixed platform but also on a mobile platform that carries out traffic in various environments. Landing UAVs on a service charging station is realized with the help of various systems of navigation and analysis of the surrounding area. The paper [8] describes the vision system developed for target detection and tracking. Adopted an optimized solution to save computation time ensuring a real time image processing supported by the robot operating system environment. The vision system works in indoor / outdoor environments even in absence of GPS or similar localization systems.

A new decentralized method for controlling the joint functioning of a UAV and a mobile platform was considered in [9]. The presented experimental results for the small Aeryon Scout and the Clearpath Robotics A200 Husky mobile platform confirm the possibility of landing both in enclosed spaces with high-quality navigation data and outdoors in windy conditions.

In the paper [10] proposes a vision-based target following and landing system for a quadrotor vehicle on a moving platform. The system employs a vision based landing site detection and locating algorithm using an omnidirectional lens. The latest smartphone was attached to the UAV and served as an on-board image acquisition and process unit. Measurements from the omnidirectional camera are combined with a proper dynamic model in order to estimate position and velocity of the moving platform. An adaptive control scheme was implemented on the flight computer to deal with unknown disturbances in outdoor environments. The system was validated on a quadrotor UAV and the vehicle successfully landed on the moving platform during outdoor flight tests. 
In [11] present the design and implementation of a vision based autonomous landing algorithm using a downward looking camera. To demonstrate the efficacy of algorithms emulate the dynamics of the ship-deck, for various sea states and different ships using a six degrees of freedom motion platform. Present the design and implementation of robust computer vision system to measure the pose of the ship-deck with respect to the vehicle. A Kalman filter is used in conjunction with vision system to ensure the robustness of the estimates.

The paper [12] suggests analysis of unmanned ground vehicle component power requirements followed by recommendations for the use of low power sensors that do not affect vehicle functionality. Design specifications for an order of magnitude higher endurance unmanned ground vehicle compared with the existing one, based on a hybrid battery configuration. Design itself of an energy-efficient (solar cell) gimballed landing platform serving as a transport and charging base for small unmanned helicopters. An indirect benefit of this design is that it also allows the unmanned vertical takeoff and landing vehicle to take off from one unmanned ground vehicle and land on another, maximizing operational range.

To increase the autonomy of an unmanned aerial vehicle, it is necessary to recharge its power source and replenish other consumables based on automated recharge systems. For systems of UAVs to achieve near autonomy, the automation of ground tasks is required. To address this problem we first conducted an economic evaluation of two solutions concepts: refill and exchange of consumable reservoirs. We demonstrated that refilling stations are economically superior for low target coverage. For high coverage systems, exchange stations are better [13].

In work [13] three types of charging stations for the UAV are proposed: Rollin 'Mat, Concentric circles, Honeycomb. They differ in cost, features and functions. Power stations such as Rollin 'Mat and Concentric circles are simple in design, easy to integrate and have a relatively low cost. However, the location and size of the terminals at the station depend on the type of UAV, which naturally affects the size of the landing pad at the station. In particular, if the UAV is rather small, the landing accuracy provided by the navigation systems may not be sufficient for docking with the charging sites. The Honeycomb power station has many advantages the system is easily expandable, because a large number of UAVs can be charged at the same time by adding additional elements and chargers. Another feature is that the wireless IR emitter/sensor communication system can be readily replaced with another wireless system. Honeycomb can be used in almost any situation, when you need to recharge. But this solution is more expensive, therefore the Honeycomb platform is recommended in case when exact landing is required on a small site in difficult weather conditions.

Also [13] proposed a system for changing the UAV battery. Using an exchange process could increase significantly the ratio of maximum possible flight time (from take-off to landing) per ground time, therefore, decreasing the total number of UAVs. On the other hand, implementation cost will increase, since exchanging an empty reservoir for a full one is a more sophisticated approach than simply charging a waiting UAV.

The paper [14] presented designs options for an autonomous UAV battery replacement system ground station. Station is to automatically swap the depleted batteries of a UAV for a recharged battery without human intervention. Here, instead of tackling navigation control algorithm problems, we work with design parameters to solve issues such as: guiding the UAV to the battery replacement site, orienting the UAV in a desired direction, locking the UAV position on the station, UAV-battery connections: extracting and placing a battery in the UAV, Battery transportation and Battery array recharging.

The design options presented in [14] assume that the ground stations are outdoors, where weather conditions cannot be predicted. The focus here is not on landing algorithms, but on how to address UAV positioning after landing with small error. While UAV navigation systems are assumed to exist, allow landing position error. The goal is to allow the UAV to reach the location where battery swapping will be conducted, even if its landing position is non ideal due to navigation errors, weather conditions, UAV damage, etc. Normally, batteries are connected to UAVs via wire plugs and placed inside the $\mathrm{UAV}$, thereby, ensuring terminal connection and battery stability. However, in a platform that has to rapidly swap a UAV's depleted battery for a recharged one, such a connection/holding system is not suitable. The method by which the battery is secured and physically connected to the UAV is of great importance because it will effect the repeatability, complexity, and the time that the ground station will take to swap batteries. Also, the added weight will effect the UAV's payload and flight time. In order to create the interface between UAV and platform, mechanical and magnetic couplers are considered and devices that can easily hold and release batteries while ensuring terminal connections in a UAV are proposed.

When the service station functions, [14] uses several modules to replace the batteries with high accuracy: orientation-fixing module, UAV Locking/unlocking module, battery extraction module, battery swap module. The station can compensate for the orientation and positioning errors of the UAV during landing. The complete station is able to compensate for orientation and positioning errors. It addresses navigation imprecision, weather conditions and/or UAV damage, which are common issues in outdoor missions and combat environments. The ground station is also capable of handling heterogeneous UAV fleets with not only different shapes and sizes, but also different number of battery cells per battery pack.

For the recharge platform, the duration the time UAV spends at the service station should be at least as long as a battery charging time; the UAV remains with the platform until its battery is fully charged. For the replacement platform the time UAV spends at the service station will be small relative to the charging time a battery requires to achieve full charge when fully depleted [14].

Figure 1 presents a classification of robotic battery service systems for multi-copter batteries. The battery service systems can be divided into the following 
features: 1) by type of basing: ground and water surface; 2) by the way of navigation during landing: local navigation systems, vision systems and global navigation systems; 3) by form of a landing site: flat, toroidal, system with mechanical guides to the center of the platform; 4) by method of battery recovery: battery charge system, battery replacement system. In the battery charge system there are two types: contact and non-contact. In the battery replacement system, depending on the way the battery is attached, there are magnetic and mechanical systems; by the type of placement of batteries there are linear and disc stores.

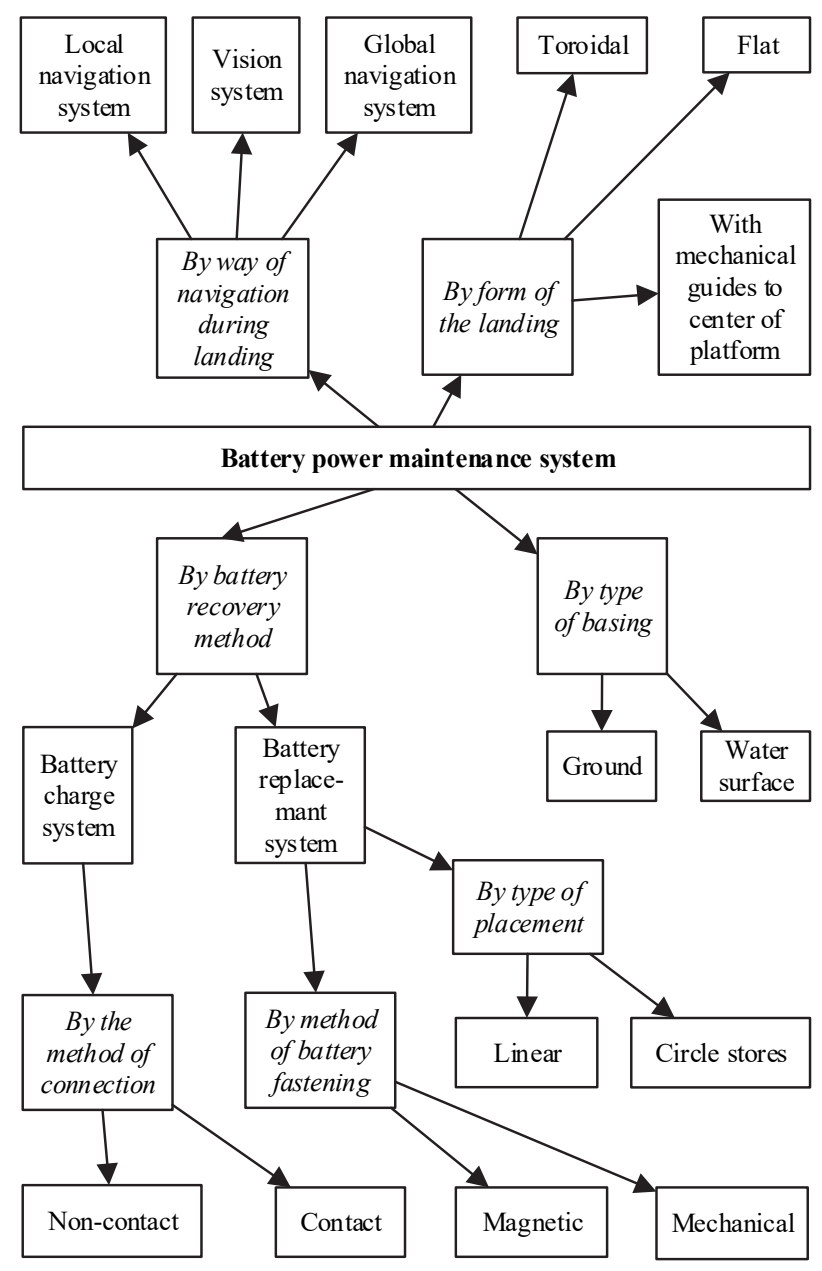

Fig. 1. Classification of robotic battery service systems for multi-copter batteries.

The design of battery maintenance systems for multicopters with battery charge is simpler and less expensive. At the same time, the speed of service is not great and the number of simultaneously charged multicopters is limited. Systems with battery replacement have more complicated design, but the speed of their maintenance is much faster, and therefore the number of overcharged multi-copters also increases.

With the development of wireless charging technology and battery replacement service stations, the UAV developed an algorithmic model for the interaction between multi-copter and service ground robotic station. Figure 2 shows the algorithmic model of the battery service systems when solving the target agrarian problem. The first step is monitor the current battery charge of UAV. If the battery is discharged, the UAV flies to the platform.

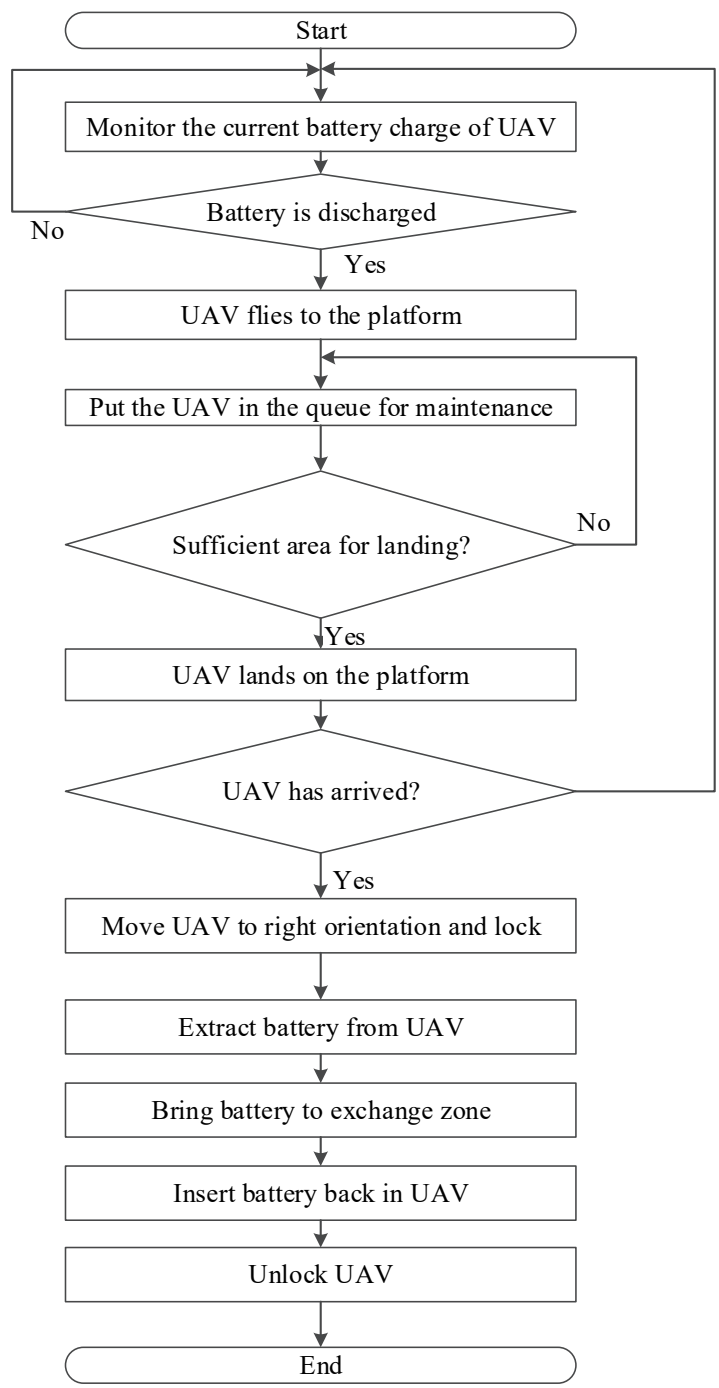

Fig. 2. Algorithm for servicing the UAV on terrestrial service platform.

The next step is to put the UAV in the queue for maintenance in accordance with the current battery charge. After receiving the charging requirement signal from the UAV, the system notifies the UAV of the presence of sufficient area for landing. Upon receiving the signal, the UAV lands on the platform with an acceptable error. After landing, the system positions drag UAV to the desired position and locks the UAV. Follows the step of extracting the battery from the UAV. The discharged battery is removed from UAV and moved to the charging area, the full battery is inserted back in UAV. Next UAV is unlocked and take off.

\section{Conclusion}

Now unmanned aerial vehicles are actively used for monitoring land, mapping the yields of land, planning zones for fertilizer application. Already the first prototypes appear, physically interacting with surrounding objects, which requires the consumption of 
even greater energy resources. The joint work of robotic ground platforms and multi-copters allows significantly increase the duration of work in the autonomous mission [15-18].

The article analyzes the requirements of the UAV battery maintenance systems for three main target areas: entertainment, farming, security. Analyzing the requirements and limitations of UAV battery charging stations. They were distinguished in the speed of processing and the complexity of the servicing mechanism.

Robotic systems for servicing power supply modules UAV can be divided into two main categories: battery charge system, battery replacement system. The battery replacement system have more complex designs, but provide a high speed of service, which increases the number of UAVs that have replenished their energy resources.

The algorithm of UAV service on a terrestrial service platform is proposed, which includes the main stages of interaction between UAV and service ground robotic station when solving the target agrarian problem.

The most promising method of battery recovery is wireless methods. The main problem of existing commercially available solutions for wireless transmission systems is low transmission power. The battery capacity of autonomous robots far exceeds the capacity of power sources for smartphones and other low-power devices whose extensive market dictates requirements to developers and manufacturers. Charging robotic systems requires capacities that are tens of times greater than chargers of existing standards. As future work, the development and prototyping of wireless charging station for UAV will be carried out.

This work was supported by RFBR № 18-5876001_ERA.Net.

\section{References}

1. O.A. Lipa, L.V. Belyaeva, S.I. Kopylov, E.V. Khromov, Questions of electromechanics. 144, 1620 (2015)

2. V.A. Prince, B.V. Vishnyakov, Yu.V. Vizilter, V.S. Gorbatsevich, O.V. Vygolov, SPIIRAS Proceedings, 45, 26-44 (2016)

3. V.A. Bogushevskaya, et al., MAI Proceedings, 51 (2012)

4. P.F. Kemper, K.A.O. Suzuki, J.R. Morrison, Journal of Intelligent \& Robotic Systems, 61, 369-397 (2011)

5. V. Nguyen, Q. Vu, O. Solenaya, A. Ronzhin, 12th International Scientific-Technical Conference on Electromechanics and Robotics "Zavalishin's Readings" - 2017, MATEC Web of Conferences, 113 (02009) (2017)

6. Q. Vu, V. Nguyen, O. Solenaya, A. Ronzhin, International Conference on Interactive Collaborative Robotics, 10459, 260-267 (2017)
7. A.S. Kodyakov, N.A. Pavlyuk, V.Yu. Budkov, R.A. Prakapovich, Stability Study of Anthropomorphic Robot Antares under External Load Action. IOP Conf. Series: Journal of Physics: Conf. Series, 803, 012074 (2017).

8. F. Cocchioni et al., Journal of Intelligent \& Robotic Systems, 84, 511-528 (2016).

9. J.M. Daly, Y. Ma, S.L. Waslander, Autonomous Robots, 38, 179-191 (2015)

10. J.W. Kim, Y.D. Jung, D.S. Lee, D.H. Shim, Journal of Intelligent \& Robotic Systems, 84, 529-541 (2016)

11. Sanchez-Lopez J. L., Pestana J., Saripalli S., Campoy P. An Approach Toward Visual Autonomous Ship Board Landing of a VTOL UAV. Journal of Intelligent \& Robotic Systems, 74, 113127 (2014)

12. S. Ioannou, K. Dalamagkidis, K.P. Valavanis, E.K. Stefanakos, Journal of Intelligent \& Robotic Systems, 53, 399-416 (2008)

13. P.F. Kemper, K.A.O. Suzuki, J.R. Morrison, Journal of Intelligent \& Robotic Systems, 61, 369-397 (2011)

14. K.A.O. Suzuki, P.K. Filho, J.R. Morrison, Journal of Intelligent \& Robotic Systems, 65, 563-586, (2012)

15. A. Zalevsky, O. Osipov, R. Meshcheryakov, International Conference on Interactive Collaborative Robotics, 268-274 (2017)

16. N. Pavluk, A. Ivin, V. Budkov, A. Kodyakov, A. Ronzhin, Interactive Collaborative Robotics, 113123 (2016)

17. A. Ronzhin, I. Vatamaniuk, N. Pavliuk, 2016 International Conference and Exposition on Electrical and Power Engineering (EPE), 675-680 (2016)

18. A.A. Ivanov, O.A. Shmakov, SPIIRAS Proceedings. 49 (6), 190-207 (2016) 